\title{
A BRIDGE BETWEEN COMPLEX GEOMETRY AND RIEMANNIAN GEOMETRY
}

\author{
ANTONIO CASSA
}

(Communicated by Jonathan M. Rosenberg)

\begin{abstract}
Every complex manifold $M^{n}$ with holomorphic metric can be obtained (at least locally) from a complex manifold $E^{2 \cdot n-2}$ and one of its $\mathbb{C}$ principal bundles $L$. The manifold $E$ is made of all (signed) complex geodesics of $M$ and $L$ is the bundle on $E$ of all choices of "times" evolving along the geodesics.
\end{abstract}

\section{INTRODUCTION}

A theory of complex manifolds with holomorphic metric has flourished within the twistor theory (cf. [BM, EL1, EL2, IYG, L1-L5, M, P, PW, W]); in this context an important place is held by LeBrun's result (cf. [L1]), which shows how, at least locally, every $n$-dimensional complex manifold with holomorphic conformal structure can be obtained starting from a complex manifold of dimension $2 \cdot n-3$ without any other structures.

The aim of this article is to show that it is possible to enrich that result: every $n$-dimensional complex manifold $(M, g)$ provided with a holomorphic metric can be obtained, at least locally, starting from a complex manifold $E$ of dimension $2 \cdot n-2$ and from one of its principal $\mathbb{C}$-bundles $\beta: L \rightarrow E$.

In a sense, which we shall define exactly further on, the manifold $E$ is the manifold of all complex "geodesics" in $M$ and the problem lies in being able to distinguish in $E$ those subsets made of "geodesics" coming out from each point of $M$. Actually this operation takes place in the total space of the bundle $L$, which has, as a fibre, on every "geodesic" the set of all possible choices of "time", which evolves along the "geodesic" [note that this bundle is never the product bundle (cf. the remarks following Theorem 3)].

Take a point $p$ of $M$ and set an initial "time" $u$ in $p$-this "time"evolves along all the geodesics coming out of $p$ and originates a submanifold $K_{p, u}$ of $L$; if a different initial "time" $v$ is chosen, the submanifold $K_{p, v}$ is anything but the "translation" of value $v-u$ of $K_{p, u}$ in $L$. These submanifolds $K_{p, u}$ are all copies of the standard regular $(n-1)$-quadric and are embedded in $L$ with a normal bundle of a well-prescribed type.

Received by the editors September 27, 1991.

1991 Mathematics Subject Classification. Primary 53C20, 32G10; Secondary 53C22, 53B20, 53B30, 83C99. 
In order to go back to the points we have to select all the $(n-1)$-quadrics in $L$ with that prescribed normal bundle and we obtain a manifold $C^{\wedge}$ of dimension $n+1$. In this manifold the holomorphic tangent space to a point $p^{\prime}$ (corresponding to a quadric $K$ in $L$ ) is naturally isomorphic to the space $H^{\circ}(K, \mathscr{O}(N))$ of holomorphic sections of the normal bundle $N$ on $K$. Thanks to the nature of the normal bundle the sections vanishing somewhere make a cone $\Gamma_{p^{\prime}}$, projecting a regular quadric, in $H^{\circ}(K, \mathscr{O}(N))$ and then in $T_{p^{\prime}}^{\prime}$. Moreover the action of $\mathbb{C}$ on $L$ moves to an action on $C^{\wedge}$ and defines a holomorphic tangent vector field $A$ on $C^{\wedge}$; if the points where $A_{p^{\prime}}$ is in $\Gamma_{p^{\prime}}$ are discarded, we get an open subset $C$ of $C^{\wedge}$ and for every $p^{\prime}$ in $C$ it is possible to find in $T_{p^{\prime}}^{\prime}$ one and only one metric tensor $g_{p^{\prime}}$ having $\Gamma_{p^{\prime}}$ as the cone of null vectors and with quadratic norm -1 on $A_{p^{\prime}}$.

Since for every point in $M$ there are infinite quadrics in $L$, we must identify in $C$ the points along the integral curves of the field $A$ and finally we obtain a manifold $R$ of dimension $n$ that "contains" the points of $M$. The metric in $p=\left[p^{\prime}\right]$ is achieved identifying the space $T_{p}^{\prime} R$ with the subspace $T_{p^{\prime}}^{\prime} C$ orthogonal to $A_{p^{\prime}}$.

The procedure described above can be applied to every complex manifold $E$ (of even dimension greater than 3 ) and to each of its principal $\mathbb{C}$-bundles, and it is effective enough to produce every complex manifold (of dimension greater than 2) with holomorphic metric and geodesically convex (therefore, at least locally, every holomorphic riemannian manifold).

So far we have referred to the space $E$ as the space of complex "geodesics" of $M$, actually the elements of $E$ are more precisely "signed" geodesics, that is, geodesic curves of $M$ along which a "signed" tangent vector evolves autoparallely (a "signed" tangent vector is a tangent vector $X$ with a chosen root of $g(X, X))$. Therefore in $E$ there are, in general, two "signed" geodesics for every nonnull geodesic of $M$ and only one "signed" geodesic for every null geodesic of $M$.

Notation.

$$
\begin{aligned}
\mathbf{Q}(M)= & \text { quadric bundle on } M \text { of null directions for }(M, g), \\
\mathbf{N}(M)= & \text { space of null geodesics of } M \\
\mathbf{Y}(F)= & \text { space of normal quadrics in } F \\
\mathbf{D}(M)= & \mathbf{Y}(\mathbf{N}(M)) \\
\mathbf{C}(B, F)= & \text { space of transverse normal quadrics in } F \\
\mathbf{R}(B, F)= & \text { space of } F \text {-normal quadrics in } B=\text { space of } \\
& \text { classes of transverse normal quadrics in } F \\
\mathbf{G}(M)= & \text { space of geodesics of }(M, g), \\
\mathbf{E}(M)= & \text { space of signed geodesics of }(M, g), \\
\mathbf{L}(M)= & \text { C-bundle on } \mathbf{E}(M) \text { of choices of time } \\
& \text { for signed geodesics. }
\end{aligned}
$$

Denoting by $\mathbf{Q}^{m}$ the regular $m$-dimensional quadric of $\mathbf{P}^{m+1}$ we will consider as in LeBrun (cf. [L1, III. 2]) on $\mathbf{Q}$ the $(m+1)$-vector bundle $\mathbf{T}^{\prime} \mathbf{P}_{\mid \mathbf{Q}}^{m+1} \otimes \mathbf{H}^{*}$. 
1. Definition. A normal $m$-quadric in a manifold $F$ is an $m$-quadric holomorphically embedded in $F$ with normal bundle $\mathbf{T}^{\prime} \mathbf{P}_{\mid \mathbf{Q}}^{m+1} \otimes \mathbf{H}^{*}$. A normal quadric $\mathbf{Q}$ is transverse with respect to the action of a 1-parameter group of biholomorphisms of $F$ if all the integral curves coming out from points of $\mathbf{Q}$ are not tangent to $\mathbf{Q}$.

2. Definition. Let $B$ be a complex manifold of even (complex) dimension $2 \cdot m$ (with $m \geq 2$ ) and let $\beta: F \rightarrow B$ be a holomorphic principal $\mathbb{C}$-bundle on $B$; an immersed $m$-quadric of $B$ is called $F$-normal if it is the image on $B$ of a transverse normal $m$-quadric of $F$.

3. Theorem. Let $B$ be a complex manifold of even (complex) dimension $2 \cdot m$ (with $m \geq 2$ ) and let $\beta: F \rightarrow B$ be a holomorphic principal $\mathbb{C}$-bundle on $B$; the space $R=\mathbf{R}(B, F)$ of all $F$-normal quadrics of $B$ has a natural structure of an $(m+1)$-dimensional holomorphic riemaniannan manifold.

Proof. Let us denote by $C^{\wedge}$ the $(m+2)$-dimensional manifold of all normal $m$-quadrics $\mathbf{Q}_{p^{\prime}}$ of $F$; the holomorphic tangent space $T_{p^{\prime}}^{\prime} C^{\wedge}$ to a point $p^{\prime}$ of $C^{\wedge}$ is isomorphic to $H^{0}\left(\mathbf{Q}_{p^{\prime}}, \mathscr{O}(N)\right)$, where $N$ is the normal bundle of $\mathbf{Q}_{p^{\prime}}$ in $F$. The space $C^{\wedge}$ has a natural holomorphic conformal structure $\Xi$ (cf. [L1, Theorem III. 2]) defined by the bundle of regular quadrics in $\mathbf{P} T^{\prime} C^{\wedge}$ given by assigning for every $p^{\prime}$ in $C^{\wedge}$ the cone corresponding in $T_{p^{\prime}}^{\prime} C^{\wedge}$ to the set $\left\{s \in H^{0}\left(\mathbf{Q}_{p^{\prime}}, \mathscr{O}(N)\right): s\right.$ has a zero $\}$. The action of $\mathbb{C}$ on $F$ induces an action $\alpha_{z}$ of $\mathbb{C}$ on $C^{\wedge}$; since for every compact subset $K$ of $F$ the number $\sup \left\{\operatorname{diam}\left(K \cap F_{b}\right): b \in B\right\}$ is finite, for every compact subset $H$ of $C^{\wedge}$ the number $r(H)=\sup \left\{|z|: \alpha_{z}(H) \cap H \neq \varnothing\right\}$ is also finite and $\alpha_{z}\left(\mathbf{Q}_{p^{\prime}}\right)=\mathbf{Q}_{p^{\prime}}$ in $F$ only for $z=0$ (the group $\left\{z \in \mathbb{C}: \alpha_{z}\left(\mathbf{Q}_{p^{\prime}}\right)=\mathbf{Q}_{p^{\prime}}\right\}$ is limited in $\mathbb{C}$ ). Denoting by $A$ the field on $C^{\wedge}$ defined by the action of $\mathbb{C}$, let us denote by $C=\mathbf{C}(B, F)$ the open subset of $C^{\wedge}$ of transverse quadrics, this is exactly the set where $A$ is not a null vector. On $C$ there is a (unique) holomorphic metric $g^{\prime}$ defining $\Xi$, invariant by the action of $\mathbb{C}$ and with quadratic norm -1 (or any other fixed nonzero complex number) on all the vectors of the field $A$. In fact, since $A$ is never zero on $C$, for every point $p_{0}^{\prime}$ of $C$ it is possible to find an open neighborhood $V^{\prime}$ biholomorphic with a polydisc of $\mathbb{C}^{m+2}$ where the action $\alpha_{z}$ in $C$ becomes the sum of $z$ in the last coordinate and where, moreover, $\Xi$ is defined by a holomorphic metric $g^{\prime \prime}$. The field $A$ is a conformal field for $g^{\prime \prime}$, rescaling the metric we can make it a Killing field: there is a holomorphic function $f\left(z, p^{\prime}\right)$ in a neighborhood of $\left(0, p_{0}^{\prime}\right)$ such that $\alpha_{z}^{*} g^{\prime \prime}=\exp \left[f\left(z, p^{\prime}\right)\right] \cdot g_{p^{\prime}}^{\prime \prime} ;$ taking $h$ such that $A_{p^{\prime}}(h)=(\partial f / \partial z)\left(0, p^{\prime}\right)$ the new metric $g^{\prime}$ defined by $g_{p^{\prime}}^{\prime}=\exp \left(-h\left(p^{\prime}\right)\right) \cdot g_{p^{\prime}}^{\prime \prime}$ has $L_{A} g^{\prime}=0$ (its coefficients do not depend on the last coordinate). Normalizing $g^{\prime}$ on the field $A$ we get a metric with the desired properties, that is, the unique one in the conformal class. The unicity implies that these local metrics patch together giving a global metric of $C$. In $C$ it is therefore possible to find a base $\mathbb{V}^{\prime}=\left\{V_{j}^{\prime}\right\}_{j \in J}$ of the above coordinate open subsets where, moreover, two points are equivalent under the action of $\alpha$ if and only if they equal all the coordinates except, at most, the last one. Otherwise near a point $p_{0}^{\prime}$ of $C$ it would be possible to find two sequences of points $\left\{p_{n}^{\prime}\right\},\left\{p_{n}^{\prime \prime}\right\}$ converging to a $p_{0}^{\prime}$ with $p_{n}^{\prime \prime}=\alpha_{z(n)}\left(p_{n}^{\prime}\right)$ but such that not all the first $m+1$ coordinates of $p_{n}^{\prime}$ and $p_{n}^{\prime \prime}$ are equal; we would have at the same time $|z(n)| \geq \varepsilon>0$ and $|z(n)| \leq r\left(\left\{p_{0}^{\prime}\right\} \cup\left\{p_{n}^{\prime}, p_{n}^{\prime \prime}\right\}\right)$ and, 
therefore, there would exist a complex number $z \neq 0$ limit point for $\{z(n)\}$ such that $\alpha_{z}\left(p_{0}^{\prime}\right)=p_{0}^{\prime}$, which is false.

Let us consider now the quotient space $R^{\prime}$ of all orbits of $C$ via the action of $\mathbb{C}$ and the quotient map $q: C \rightarrow R^{\prime}$. The map $\sigma: R^{\prime} \rightarrow R$ defined by $\sigma\left(\left[Q^{\prime}\right]\right)=\beta\left(Q^{\prime}\right)$ is well defined and surjective. It is also injective because $\beta\left(Q^{\prime}\right)=\beta\left(Q^{\prime \prime}\right)$ implies there is a $z$ in $\mathbb{C}$ such that $Q^{\prime \prime}=\alpha_{z}\left(Q^{\prime}\right)$. In fact, the set $X=\left\{z \in \mathbb{C}: \alpha_{z}\left(Q^{\prime}\right) \cap Q^{\prime \prime} \neq \varnothing\right\}$ is an analytic subset of $\mathbb{C}$ contained in the disk of center 0 and radius $r\left(Q^{\prime} \cup Q^{\prime \prime}\right)$ and, therefore, is a finite set $X=\left\{z_{1}, \ldots, z_{k}\right\}$; for at least one (and only for one) of these complex numbers $z_{j}$ the intersection $Q^{\prime} \cap \alpha_{-z(j)}\left(Q^{\prime \prime}\right)$ has a nonempty interior, for that number we have $Q^{\prime \prime}=\alpha_{z(j)}\left(Q^{\prime}\right)$. We will identify the space $\mathbf{R}(B, F)$ with $R^{\prime}$ via the map $\sigma$.

The space $R^{\prime}$ is a Hausdorff topological space; in fact, if $\left\{p_{n}^{\prime}\right\},\left\{p_{n}^{\prime \prime}\right\}$ are nets of equivalent points of $C$ with $p_{n}^{\prime \prime}=\alpha_{z(n)}\left(p_{n}^{\prime}\right)$ and converging to $p^{\prime}, p^{\prime \prime}$ we have $|z(n)| \leq r\left(\left\{p_{n}^{\prime}, p_{n}^{\prime \prime}\right\} \cup\left\{p^{\prime}, p^{\prime \prime}\right\}\right)$ and, therefore, $p^{\prime \prime}=\alpha_{z}\left(p^{\prime}\right)$ where $z$ is a complex number limit point for the net $\{z(n)\}$. For every chart $\left(V^{\prime}, \varphi^{\prime}\right)$ in $\mathbb{V}^{\prime}$ let us define $V=q\left(V^{\prime}\right)$ and $\varphi: V \rightarrow \mathbb{C}^{m+1}$ by $\varphi\left(q\left(p^{\prime}\right)\right)==\left(z^{1}\left(p^{\prime}\right), \ldots\right.$, $\left.z^{m+1}\left(p^{\prime}\right)\right)$. The map $\varphi$ is a well-defined homeomorphism between $V$ and an open subset of $\mathbb{C}^{m+1}$. The family $\mathbb{V}=\left(V_{j}\right)_{j \in J}$ is a holomorphic atlas making $R^{\prime}$ a complex manifold of dimension $m+1$.

For every $p^{\prime}$ in $C$ the set $H_{p^{\prime}}=\left\{X^{\prime} \in T_{p^{\prime}}^{\prime}: g^{\prime}\left(X^{\prime}, A\right)=0\right\}$ is a nondegenerate $(m+1)$-dimensional subspace of $T_{p^{\prime}}^{\prime}$, the map $q_{* p^{\prime}}: H_{p^{\prime}} \rightarrow T_{q\left(p^{\prime}\right)}^{\prime}$ is an isomorphism, and the metric $g$ on $R^{\prime}$ defined by $g_{q\left(p^{\prime}\right)}\left(q_{*}\left(X^{\prime}\right), q_{*}\left(Y^{\prime}\right)\right)=$ $g_{p^{\prime}}^{\prime}\left(X^{\prime}, Y^{\prime}\right)$ for $X^{\prime}, Y^{\prime}$ in $H_{p^{\prime}}$ is a well-defined holomorphic metric.

The map $q: C \rightarrow R^{\prime}$ makes $C$ a principal $\mathbb{C}$-bundle on $R^{\prime}$. In fact, taking two charts $\left(V^{\prime}, \varphi^{\prime}\right)$ and $(V, \varphi)$ as in the proof above the map $\Phi: q^{-1}(V) \rightarrow$ $V \times \mathbb{C}$ defined by $\Phi\left(\alpha_{z}\left(p^{\prime}\right)\right)=\left(q\left(p^{\prime}\right), z^{m+2}\left(p^{\prime}\right)+z\right)$ for $p^{\prime}$ in $V^{\prime}$ is well defined and is a trivialization of $C$ on $V$.

The different classes of holomorphic principal $\mathbb{C}$-bundles $F$ on $B$ are in correspondence with the elements of the space $H^{1}(B, \mathscr{O})$. The product bundle gives always $C^{\wedge}=\varnothing$; in fact, every $m$-quadric in $B \times \mathbb{C}$ is contained in a "slice" $B \times\{a\}$ and, therefore, its normal bundle contains, as a factor, the product line bundle and cannot be isomorphic with the bundle $\mathbf{T}^{\prime} \mathbf{P}_{\mid \mathbb{Q}}^{m+1} \otimes \mathbf{H}^{*}$.

When $B$ is a Stein manifold the spaces $C^{\wedge}, C$, and $R$ are empty (the only principal $\mathbb{C}$-bundle on $B$ is, in this case, the product bundle); however, we will prove in the following pages (cf. Theorem 7) that the construction of the theorem above (applied to non-Stein manifolds) is able to give (at least locally) every holomorphic riemannian manifold.

4. Definition. Let $\left(V^{(n)}, g\right)$ be a finite-dimensional (complex) vector space with a (complex) scalar product; a signed direction of $V$ is an element $l$ of the dual universal bundle $\delta: \mathbf{U}^{*} \rightarrow \mathbf{P} V$ on $\mathbf{P} V$ such that $[l(X)]^{2}=g(X, X)$ for all $X$ in the direction $\delta(l)$.

The manifold $S V$ of all signed directions of $(V, g)$ is isomorphic to the regular $(n-1)$-quadric of null directions of $V \times \mathbb{C}$ (with scalar product $\langle(X, A),(Y, B)\rangle=g(X, Y)-A \cdot B)$ via the map $\nu: \mathbf{S} V \rightarrow \mathbf{P}(V \times \mathbb{C})$ defined by $\nu(l)=[X, l(X)]$. The holomorphic map $\delta \mid: \mathbf{S} V \rightarrow \mathbf{P} V$ presents $S V$ as a branched covering of $\mathbf{P} V$ with two leaves; for every nonnull direction of $V$ 
there are exactly two signed distinct directions and only one for the null directions. A signed direction gives a direction $[X]$ in $V$ and the value $l(X)$ (a complex "norm" for $X$, that is, a square root of $g(X, X))$ for every vector $X$ in that direction.

For every holomorphic riemannian manifold $\left(M^{n}, g\right)$ we will denote by $\mathbf{S} T^{\prime} M$ the bundle on $M$ of $(n-1)$-quadrics of all signed tangent directions of $(M, g)$.

There are natural holomorphic maps $\delta: \mathbf{S} T^{\prime} M \rightarrow \mathbf{P} T^{\prime} M$ and $\pi_{s}: \mathbf{S} T^{\prime} M \rightarrow$ $M$. The space $\mathbf{S} T^{\prime} M \times \mathbb{C}$ is biholomorphic to the space $\mathbf{Q}(M \times \mathbb{C})$ of null directions in the riemannian manifold $M \times \mathbb{C}$ via the map $\chi: \mathbf{S} T^{\prime} M \times \mathbb{C} \rightarrow$ $\mathbf{Q}(M \times \mathbb{C})$ defined by $\chi(l, t)=[X, l(X)]_{(\pi(l), t)}$ for $X \in \delta(l)$.

For every nonconstant (complex) geodesic $\gamma: D \rightarrow M$ defined on a connected region $D$ of the complex line, we have the following well-defined maps: $\pi \gamma: D \rightarrow \mathbf{P} T^{\prime} M$ by $\pi \gamma(t)=\left[\gamma^{\prime}(t)\right]: \sigma_{k} \gamma: D \rightarrow \mathbf{S} T^{\prime} M$ by $\sigma_{k} \gamma(t)=($ the unique linear map on $\mathbb{C} \cdot \gamma^{\prime}(t)$ with value $k$ on $\left.\gamma^{\prime}(t)\right)$; and $\sigma_{k, a} \gamma: D \rightarrow \mathbf{S} T^{\prime} M \times \mathbb{C}$ by $\sigma_{k, a} \gamma(t)=\left(\sigma_{k} \gamma(t), k \cdot t+a\right)$ where $k, a$ are complex numbers with $k^{2}=$ $g\left(\gamma^{\prime}(t), \gamma^{\prime}(t)\right)$.

The curves $\pi \gamma, \sigma_{k} \gamma$, and $\sigma_{k, a} \gamma$ foliate $\mathbf{P} T^{\prime} M, \mathbf{S} T^{\prime} M$, and $\mathbf{S} T^{\prime} M \times \mathbb{C}$, respectively.

For every nonzero tangent vector $X$ in $\mathbf{T}_{p}^{\prime} M$ there is an (inextensible) complex geodesic $\gamma_{x}: D_{x} \rightarrow M$ such that $\gamma_{x}\left(z_{0}\right)=p$ and $\gamma_{x}^{\prime}\left(z_{0}\right)=X$ for some $z_{0}$ in $D_{x}$.

5. Definition. The space of leaves of the foliation of $\mathbf{P} T^{\prime} M$ is the space $\mathbf{G}(M)=\mathbf{G}(M, g)$ of geodesics of $M$, the space of leaves of $S T^{\prime} M$ will be called the space $\mathbf{E}(M)=\mathbf{E}(M, g)$ of signed geodesics of $M$; and the space of leaves of $\mathbf{S} T^{\prime} M \times \mathbb{C}$ will be called the space $\mathbf{L}(M)=\mathbf{L}(M, g)$ of choices of time on the signed geodesics.

There is a natural map $\beta: \mathbf{L}(M) \rightarrow \mathbf{E}(M)$ defined by $\beta([l, t])=[l]$ and a continuous action of $\mathbb{C}$ on $\mathbf{L}(M)$ given by $\alpha_{z}([l, t])=[l, t+z]$ such that $\mathbb{C} \cdot[l, t]=\beta^{-1}([l])$. The map $\delta: \mathbf{S} T^{\prime} M \rightarrow \mathbf{P} T^{\prime} M$ induces a map $\delta^{\wedge}: \mathbf{E}(M) \rightarrow$ $\mathbf{G}(M)$.

Since $\chi \circ \sigma_{k, a} \gamma$ is the lifting to $\mathbf{Q}(M \times \mathbb{C})$ of the null geodesic $(\gamma(u), k \cdot u+a)$ in $M \times \mathbb{C}$, the map $\chi$ induces a homeomorphism $\chi^{\wedge}$ between $\mathbf{L}(M)$ and the space $\mathbf{N}(M \times \mathbb{C})$ of null geodesics in $M \times \mathbb{C}$.

A justification for the name given to the bundle $\mathbf{L}(M)$ is: let $\sigma_{1} \gamma_{x}$ be a signed geodesic ran with unitary speed $(g(X, X)=1)$ reaching the signed direction $l$ for the value $z$ of the parameter $\left(\sigma_{1} \gamma_{x}(z)=l\right)$; if we decide to assign to $l$ the "initial time" $u$ it is natural to say that the time "evolves" along the geodesic becoming $z^{\prime}-z+u$ in $l^{\prime}=\sigma_{1} \gamma_{x}\left(z^{\prime}\right)$. Therefore a choice of time for a (nonnull) signed direction is a couple $(l, u)$ in $\mathbf{S} T^{\prime} M \times \mathbb{C}$ and two of such choices $(l, u),\left(l^{\prime}, u^{\prime}\right)$ are equivalent if and only if there is a tangent vector $X$ with $g(X, X)=1$ and two values $z, z^{\prime}$ in the domain of definition of $\sigma_{1} \gamma_{x}$ such that $\sigma_{1} \gamma_{x}(z)=l, \sigma_{1} \gamma_{x}\left(z^{\prime}\right)=l^{\prime}$, and $u^{\prime}-u=z^{\prime}-z$. This is the same as to claim that $(l, u)$ and $\left(l^{\prime}, u^{\prime}\right)$ are joined by a curve $\sigma_{k, a} \gamma$ in $\mathbf{S} T^{\prime} M \times \mathbb{C}$.

For null signed directions it is reasonable to call equivalent $(l, u)$ and $\left(l^{\prime}, u^{\prime}\right)$ if and only if $l$ and $l^{\prime}$ are joined by a signed geodesic in $\mathrm{S} T^{\prime} M$ and $u=u^{\prime}$ (the time "stops" on null geodesics); again this is equivalent to the claim that $(l, u)$ and $\left(l^{\prime}, u^{\prime}\right)$ are joined by a curve $\sigma_{k, a} \gamma$. 
6. As in [L1] a manifold $M$ with holomorphic connection is called geodesically convex if it is geodesically convex with respect to the underlying smooth connection.

Proposition. Let $\left(M^{(n)}, g\right)$ be a geodesically convex holomorphic riemannian manifold, the spaces $\mathbf{G}(M), \mathbf{E}(M)$, and $\mathbf{L}(M)$ have a unique structure of complex manifolds of dimensions, respectively, $2 \cdot n-2,2 \cdot n-2$, and $2 \cdot n-1$ making the maps $q_{G}: \mathbf{P} T^{\prime} M \rightarrow \mathbf{G}(M), q_{E}: \mathbf{S} T^{\prime} M \rightarrow \mathbf{E}(M)$, and $q_{L}: \mathbf{S} T^{\prime} M \times \mathbb{C} \rightarrow$ $\mathbf{L}(M)$ holomorphic of maximal rank. Moreover, the map $\beta: \mathbf{L}(M) \rightarrow \mathbf{E}(M)$ makes $\mathbf{L}(M)$ a holomorphic principal $\mathbb{C}$-bundle on $\mathbf{E}(M)$.

Proof. Since the curves $\pi \gamma$ foliate $\mathbf{P} T^{\prime} M^{2 \cdot n-1}$, imitating the proof of Theorem III.1 of [L1] for every $\left[X_{0}\right]$, it is possible to find an arbitrarily small open neighborhood $W$ of $\left[X_{0}\right]$, an open subset $B$ of $\mathbb{C}^{2 \cdot n-2}$, a biholomorphism $\varphi$ between $W$ and an open subset of $\mathbb{C} \times B$, and a relatively compact and geodesically convex open subset $U$ of $M$ such that the leaves of $W$ are exactly the sets $\Lambda_{z}=\left(\operatorname{pr}_{B} \circ \varphi\right)^{-1}(z)$ (for $z \in B$ ) and every $\pi\left(\Lambda_{z}\right)$ is an inextensible geodesic of $U$.

A geodesic of $M$ does not decompose in $U$ since $U$ and $M$ are geodesically convex, hence the map $\theta: B \rightarrow \mathbf{G}(M)$ defined by $\theta(z)=q_{G}([X])$ for $[X] \epsilon$ $\Lambda_{z}$ is not only well defined, continuous, and open but, moreover, is injective. Therefore at every point $q_{G}\left(\left[X_{0}\right]\right)$ of $\mathbf{G}(M)$ the couple $\left(q_{G}(W), \theta^{-1}\right)$ gives a local chart of $\mathbf{G}(M)$; all these charts make $\mathbf{G}(M)$ a topological manifold; the transition functions between any two such local charts are holomorphic as can be seen by using a finite sequence of overlapping Frobenius charts. Proceeding as in [L1, Theorem III.1] it is possible to prove that $\mathbf{G}(M)$ is a Hausdorff manifold.

In the same way for every $l_{0}$ in $\mathbf{S} T^{\prime} M$ there exists an open neighborhood $W^{\wedge}$ of $l_{0}$, an open subset $B^{\wedge}$ of $\mathbb{C}^{2 \cdot n-2}$, a biholomorphism $\varphi^{\wedge}$ between $W^{\wedge}$, and an open subset of $\mathbb{C} \times B^{\wedge}$ such that the leaves of $W^{\wedge}$ are the sets $\Lambda_{z}^{\wedge}=\left(\operatorname{pr}_{B^{\wedge}} \circ \varphi^{\wedge}\right)^{-1}(z)$ for $z \in B^{\wedge}$ and the $\pi\left(\Lambda_{z}^{\wedge}\right)$ are inextensible geodesics of a relatively compact and geodesically convex open subset $U$ of $M$. Then it is possible to define maps $\theta^{\wedge}: B^{\wedge} \rightarrow \mathbf{E}(M)$ and charts $\left(q_{E}\left(W^{\wedge}\right),\left(\theta^{\wedge}\right)^{-1}\right)$ on $\mathbf{E}(M)$ in an analogous way. In the manifold $\mathbf{E}(M)$ it is possible to separate points with different images in $\mathbf{G}(M)$ since the map $\delta^{\wedge}: \mathbf{E}(M) \rightarrow \mathbf{G}(M)$ is continuous; if $\sigma_{k} \gamma_{X} \neq \sigma_{h} \gamma_{Y}$ in $\mathbf{E}(M)$ but $\pi \gamma_{X}=\pi \gamma_{Y}$ in $\mathbf{G}(M)$ then necessarily $\sigma_{h} \gamma_{Y}=\sigma_{-k} \gamma_{X}$ (with $k \neq 0, X \neq 0$ ) and it is possible to find an open neighborhood $W$ of $X$ in $T^{\prime} M$ and an open neighborhood $D$ of $k$ in $\mathbb{C}$ such that for every $X^{\prime}, Y^{\prime}$ in $W$ and every $k^{\prime}, h^{\prime}$ in $D, \sigma_{k^{\prime}} \gamma_{X^{\prime}} \neq \sigma_{-h^{\prime}} \gamma_{Y^{\prime}}$ holds. Otherwise there would exist two sequences $\left\{X_{n}\right\},\left\{Y_{n}\right\}$ converging to $X$ in $T^{\prime} M$, two sequences $\left\{k_{n}\right\},\left\{h_{n}\right\}$ converging to $k$ in $\mathbb{C}$, and a sequence $\left\{\lambda_{n}\right\}$ in $\mathbb{C}^{*}$ and a sequence $\left\{z_{n}\right\}$ in $\mathbb{C}$ such that $\left(Y_{n},-h_{n}\right)=\lambda_{n} \cdot\left(\gamma_{X_{n}}^{\prime}\left(z_{n}\right), k_{n}\right)$ for every $n \geq 1$. Then taking subsequences, if necessary, it would be possible to suppose $\left\{\lambda_{n}\right\}$ converging to $\lambda \neq 0$ and $\left\{z_{n}\right\}$ converging to $z$ in $D_{x}$ and to obtain, going to the limit, $(X,-k)=\lambda \cdot(X, k)$. But this would imply $\lambda=-1$ and $X=0$.

Given a holomorphic map $\varepsilon^{\wedge}: B^{\wedge} \rightarrow\left(T^{\prime} M-0\right) \times \mathbb{C}$ with $\varepsilon^{\wedge}(z)=$ $(X(z), k(z))$ such that $[k(z)]^{2}=g(X(z), X(z))$ and $\sigma_{k(z)} \gamma_{X(z)}=\Lambda_{z}^{\wedge}$, the map $\lambda^{\wedge}: B^{\wedge} \times \mathbb{C} \rightarrow q_{L}\left(W^{\wedge} \times \mathbb{C}\right)$ defined by $\lambda^{\wedge}(z, a)=q_{L}\left(\sigma_{k(z)} \gamma_{X(z)}(u), k(z) \cdot u+a\right)$ 
is a well-defined biholomorphism and its inverse gives coordinate functions to $q_{L}\left(W^{\wedge} \times \mathbb{C}\right)$.

Since every geodesic in $M$ is injective, $\alpha_{z}([l, t])=[l, t]$ only for $z=0$. Moreover, $\beta^{-1}\left(\theta^{\wedge}\left(B^{\wedge}\right)\right)=\lambda^{\wedge}\left(B^{\wedge} \times \mathbb{C}\right)$ and $\left(\theta^{\wedge} \circ \operatorname{id}_{\mathbb{C}}\right) \circ\left(\lambda^{\wedge}\right)^{-1}: \beta^{-1}\left(\theta^{\wedge}\left(B^{\wedge}\right)\right) \rightarrow$ $\theta^{\wedge}\left(B^{\wedge}\right) \times \mathbb{C}$ is a trivialization of $\mathbf{L}(M)$ on $\theta^{\wedge}\left(B^{\wedge}\right)$; this proves also that $\mathbf{L}(M)$ is a Hausdorff manifold.

7. When $M$ is geodesically convex the map $\chi^{\wedge}: \mathbf{L}(M) \rightarrow \mathbf{N}(M \times \mathbb{C})$ is a biholomorphism (note that $M \times \mathbb{C}$ is geodesically convex, therefore, by [L1, Theorem III.1], $\mathbf{N}(M \times \mathbb{C})$ is a complex manifold). For every $p \in M$ and every $u \in \mathbb{C}$ the map $\sigma_{u}: \mathbf{S} T_{p}^{\prime} \rightarrow \mathbf{L}(M)$ defined by $\sigma_{u}(l)=\left[\sigma_{l(X), u} \gamma_{X}(z)\right]=$ $\left[\sigma_{l(X)} \gamma_{X}(z), l(X) \cdot z+u\right.$ ] (with $\left.X \in \delta(l)\right)$ is an embedding of the $(n-1)$-quadric $\mathbf{S} T_{p}^{\prime}$. The map $\chi^{\wedge}$ transforms the quadric $\sigma_{u}\left(\mathbf{S} T_{p}^{\prime}\right)=K_{p, u}$ in the normal quadric $Q_{p, u}$ in $\mathbf{N}(M \times \mathbb{C})$ made of all null geodesics of $M \times \mathbb{C}$ through the point $(p, u)$. The map $j: M \times \mathbb{C} \rightarrow C(\mathbf{E}(M), \mathbf{L}(M))$ defined by $j(p, u)=$ $K_{p, u}$ is a holomorphic embedding.

Theorem. Let $(M, g)$ be a geodesically convex (Stein) holomorphic riemannian manifold with $\operatorname{dim}(M) \geq 3$. Then $(M, g)$ is isometric to a connected component of the riemannian manifold $R(\mathbf{E}(M), \mathbf{L}(M))$.

Proof. The manifold $M \times \mathbb{C}$ with the product metric $g((X, A),(Y, B))=$ $g(X, Y)-A \cdot B$ verifies all the hypotheses of Theorem III.5 of [L1], therefore the map $j$ defined above gives a conformal embedding of $M \times \mathbb{C}$ in a connected component of $C^{\wedge}(\mathbf{E}(M), \mathbf{L}(M))=\mathbf{Y}(\mathbf{L}(M)) \simeq \mathbf{Y}(\mathbf{N}(M \times \mathbb{C}))=\mathbf{D}(M \times \mathbb{C})$ (in fact, in a connected component of $C(\mathbf{E}(M), \mathbf{L}(M)))$; the product metric $g$ of $M \times \mathbb{C}$, via $j$, becomes a holomorphic metric on $j(M \times \mathbb{C})$ invariant by the action of $\mathbb{C}$, defining the conformal structure of $C(\mathbf{E}(M), \mathbf{L}(M))$ and with quadratic norm -1 on the field $A$ generated by the action of $\mathbb{C}$. By the local unicity of such a metric (cf. the proo: of Theorem 3), the map $j$ is in fact a metric embedding of $M \times \mathbb{C}$ in $C(\mathbf{E}(M), \mathbf{L}(M))$. Then, passing to the quotient spaces of orbits given by the action of $\mathbb{C}, M$ is isometric to a connected component of $R(\mathbf{E}(M), \mathbf{L}(M))$.

\section{REFERENCES}

[BM] R. Baston and L. Mason, Conformal gravity, the Einstein equation and spaces of complex null geodesics, Classical Quantum Gravity 4 (1987), 815-826.

[EL1] M. Eastwood and C. LeBrun, Thickenings and supersymmetric extensions of complex manifolds, Amer. J. Math. 108 (1986), 1177-1192.

[EL2] _ _ Fattenings complex manifolds, J. Geom. Phys. 8 (1992), 123-147.

[IYG] J. Isenberg, P. Yassin, and P. S. Green, Non-self-dual gauge fields, Phys. Lett. B 78 (1978), $462-464$.

[L1] C. LeBrun, Spaces of complex null geodesics in complex Riemannian geometry, Trans. Amer. Math. Soc. 278 (1983), 209-231.

[L2] $\quad$ The first formal neighborhood of ambitwistor space for curved spaces, Lett. Math. Phys. 6 (1982), 345-354.

[L3] _ _ Ambitwistors and Einstein equations, Classical Quantum Gravity 2 (1985), 555-563.

[L4] _ Thickenings and gauge fields, Classical Quantum Gravity 3 (1986), 1039-1059.

[L5] _ Thickenings and conformal gravity, Comm. Math. Phys. 139 (1991), 1-43.

[M] Yu. Manin, Gauge field theory and complex geometry, Springer-Verlag, Berlin, 1988. 
[P] R. Penrose, Non linear gravitons and curved twistor theory, Gen. Relativity Gravitation 7 (1976), 31-52.

[PW] R. Penrose and R. S. Ward, Twistors for flat and curved space-time, Gen. Relativity Gravitation, One hundred years (A. Held, ed.), Plenum, New York, 1980.

[W] E. Witten, An interpretation of classical Yang-Mills theory, Phys. Lett. B 77 (1978), 394-397.

ViA Del PONTE SOSPESO 26, 50142 Firenze, ITALY 